\title{
The Smc5/6 Complex: More Than Repair?
}

\author{
A. KEGEL AND C. SJÖGREN \\ Department of Cell and Molecular Biology, Karolinska Insitutet, 17177 Stockholm, Sweden \\ Correspondence: camilla.sjogren@ki.se
}

\begin{abstract}
Through its functions in chromosome replication, segregation, and repair, the Smc5/6 complex has a central role in the maintenance of genome stability. The complex is part of the family of structural maintenance of chromosome protein complexes that also includes cohesin and condensin. Mutations in any of these complexes disrupt chromosome segregation and render cells hypersensitive to different types of DNA damage. The chromosome mis-segregation phenotypes in cohesin and condensin mutants can be attributed to their functions in sister chromatid cohesion and chromosome condensation, respectively. Cohesin-dependent chromatid cohesion is also needed for DNA double-strand break repair, whereas condensin is required for repair of single-strand breaks. How Smc5/6 promotes chromosome stability is largely unknown. Accumulating data suggest that it prevents accumulation of aberrant DNA links between sister chromatids created during repair by homologous recombination. A long-standing idea is that it also has a role in the maintenance of nondamaged chromosomes. Here, we present an overview of the current knowledge of Smc5/6 and discuss a possible nonrepair role of the complex.
\end{abstract}

Structural maintenance of chromosome (SMC) protein complexes have been implicated in many, if not all, fundamental chromosome-based processes. They regulate chromosome segregation, repair, and transcription, and recent results indicate that these multitasking complexes have an impact on chromosome replication as well (Nasmyth and Haering 2005; Hirano 2006; Terret et al. 2009; Wendt and Peters 2009). The Smc5/6 complex (Smc5/6) is no exception. It is central for correct repair by homologous recombination (HR), is needed for resolution of chromosomes during cell division, and has been implicated in a late step in replication (De Piccoli et al. 2009; Potts 2009). There are also indications that the complex is connected to regulation of gene silencing (Dhillon and Kamakaka 2000; Cuperus and Shore 2002; Zhao and Blobel 2005; Yu et al. 2010). Here, we present current knowledge of the complex's structure and give a summary of its repair functions. We also discuss the evidence for nonrepair roles of the complex. For a more detailed review on the repair function of $\mathrm{Smc5} / 6$, we refer to two recent reviews (De Piccoli et al. 2009; Potts 2009).

\section{COMPONENTS AND STRUCTURE OF THE SMC5/6 COMPLEX}

Yeast Smc5/6 consists of eight core subunits: Smc5, Smc6, and six nonstructural maintenance of chromosome element (Nse) proteins (Fousteri and Lehmann 2000; Fujioka et al. 2002; Hazbun et al. 2003; McDonald et al. 2003; Morikawa et al. 2004; Pebernard et al. 2004, 2006; Sergeant et al. 2005; Zhao and Blobel 2005). In the budding yeast Saccharomyces cerevisiae, Nse proteins are named Nse1, Mms21/Nse2, Nse3, Nse4/Qri2, Nse5, and Kre29/Nse6. In the fission yeast Schizosaccharomyces pombe, Smc6 was first identified as Rad18, Smc5 as Spr18, and Nse4 as Rad62 (Verkade et al. 1999; Fousteri and Lehmann 2000; Morikawa et al. 2004). Six of the human orthologs (hSmc5, hSmc6, hNse1, hNse2/hMms21, hNse3/MAGE1, and hNse4) have been identified and shown to form a complex in vivo (Taylor et al. 2001, 2008; Harvey et al. 2004; Potts and Yu 2005). Here, we call the subunits of the complex Smc5, Smc6, and Nse1 - Nse6. In addition to these eight core subunits, fission yeast Smc5 and Smc6 have been shown to interact with Rad60 (Boddy et al. 2003). Rad60, and its budding yeast homolog Esc2, are also functionally connected to Smc5/6 (see below), but no physical interaction between Esc 2 and the $S$. cerevisiae core complex has been reported so far.

How are the different subunits organized in the Smc5/6 complex? With the known structural features of cohesin and condensin as starting points, a picture based mostly on the yeast two-hybrid system and biochemical analysis starts to emerge (Fig. 1) (Sergeant et al. 2005; Palecek et al. 2006; Pebernard et al. 2006, 2008a; Duan et al. 2009b). SMC proteins contain a central extended $\alpha$-helical region that ends in a globular domain on each side. These globular amino and carboxyl termini include a Walker A and Walker B motif, respectively. A third globular domain is found in the middle of the protein; this is called the hinge domain because it is the region in which the SMC protein folds back on itself. This folding brings the Walker motifs of the amino and carboxyl domains together, forming an ATPase that is connected to the hinge via an antiparallel coiled coil. Both cohesin and condensin hydrolyze ATP, and the ATPase activity is needed for their function (Kimura and Hirano 1997; Arumugam et al. 2003; Weitzer et al. 2003). In vitro analysis of $S$. pombe Smc5/6 also indicates that this complex has an ATPase activity that is stimulated by double-strand DNA (Fousteri and Lehmann 2000). Furthermore, point mutations in the ATP-binding domain impair the function of Smc6 and render cells sensitive to DNA-damaging agents (Verkade et al. 1999; 
A

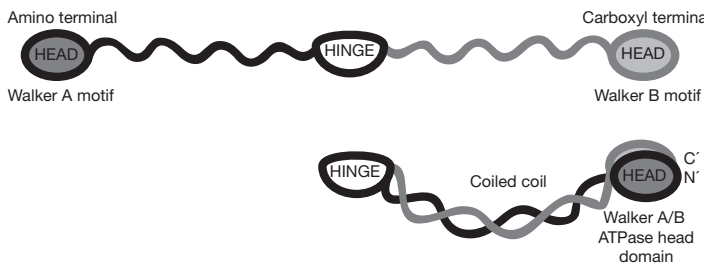

B
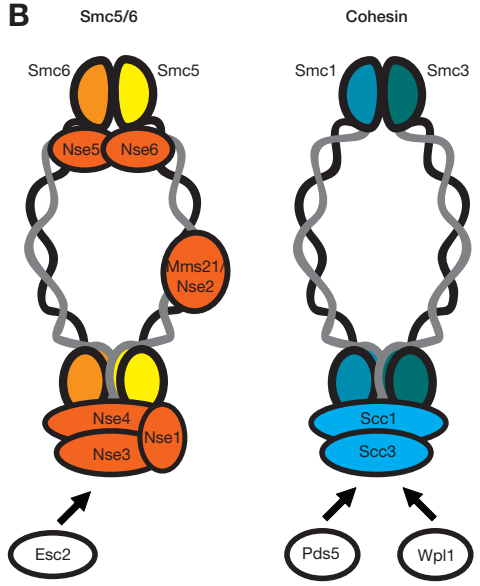

Figure 1. Structure and composition of SMC complexes. (A) An SMC protein is folded at the central hinge domain. This brings the Walker motifs in the amino- and carboxy-terminal globular domains together, creating an ATPase that is connected to the hinge domain via a long stretched coiled coil. (B) General organization of $\mathrm{Smc5} / 6$, cohesin, and condensin. Subunits are named according to the budding yeast nomenclature. With the exception of the ATPase activity of SMC proteins, only Smc5/6 contains a protein with enzymatic activity, the Nse2/Mms21 SUMO ligase. Please see text for details.

Fousteri and Lehmann 2000). Like the SMC proteins present in cohesin and condensin, Smc5 and Smc6 interact with each other via their hinge domains (Sergeant et al. 2005; Duan et al. 2009b). Nse2 binds to the coiled-coil domain of Smc5, and a recently published X-ray structure shows that this interaction is mediated by the amino-terminal domain of Nse2 (Duan et al. 2009a). Nse3, Nse4, and Nse1 form a subcomplex that associates with the globular ends of Smc5 and Smc6 (Sergeant et al. 2005; Palecek et al. 2006; Duan et al. 2009b). In the fission yeast complex, both Nse4 and Nse3 bridge Smc5 and Smc6, and Nse1 stabilizes the interaction between Nse3 and Nse4 (Sergeant et al. 2005; Palecek et al. 2006; Pebernard et al. 2008a). Nse 5 and Nse 6 have been reported to associate as a dimer near the globular heads of the fission yeast complex, but in budding yeast, this subcomplex appears to interact with the hinges of Smc5 and Smc6 (Palecek et al. 2006; Pebernard et al. 2006; Duan et al. 2009b). Whether this difference is relevant in a functional perspective, or is caused by experimental variation, remains to be established. Possibly, the divergent results are a reflection of a flexibility in the SMC arms that allows the hinge to reside in close proximity to the amino and carboxyl termini. However, it is of note that no sequence homology is shared by Nse 5 and Nse 6 from the two yeasts, and they are only essential for survival of budding, but not fission, yeast (Pebernard et al. 2006). As it stands today, the idea that Nse5 and Nse6 from the two yeasts are functional homologs is based on the observations that they are stable subunits of the Smc5/6 complex, that mutations of either lead to DNA repair defects in both yeasts, and that the two Nse6 proteins contain similar protein-folding motifs (Hazbun et al. 2003; Zhao and Blobel 2005; Pebernard et al. 2006).

\section{REPAIR FUNCTION OF THE SMC5/6 COMPLEX}

In 1995, Lehmann et al. (1995) reported that Smc6, then called Rad18, was an SMC protein needed for repair of ultraviolet (UV) and $\gamma$-ray-induced DNA damage in $S$. pombe. Today, it is known that all eight subunits of Smc5/6 are needed for DNA repair in yeast (Fujioka et al. 2002; McDonald et al. 2003; Harvey et al. 2004; Morikawa et al. 2004; Onoda et al. 2004; Pebernard et al. 2004, 2006; Hu et al. 2005; Zhao and Blobel 2005). Repair is also defective in human cells after depletion of MMS 21 by RNA interference (RNAi) or in plans carrying a nonfunctional RAD18/MIM/SMC6 gene caused by transfer-DNA (TDNA) insertion (Mengiste et al. 1999; Kozak et al. 2009). Mutations of any of the Smc5/6 subunits, including Rad60/Esc2, render cells hypersensitive to DNA-damaging agents, such as UV, ionizing irradiation, methyl methanesulfonate (MMS), and hydroxyurea (HU) (Verkade et al. 1999; Fujioka et al. 2002; Morishita et al. 2002; Harvey et al. 2004; Morikawa et al. 2004; Onoda et al. 2004; Pebernard et al. 2004, 2006, 2008a; Andrews et al. 2005; Hu et al. 2005; Sergeant et al. 2005; Torres-Rosell et al. 2005b; Zhao and Blobel 2005; Ampatzidou et al. 2006; Cost and Cozzarelli 2006; Miyabe et al. 2006; Santa Maria et al. 2007; Mankouri et al. 2009; Sollier et al. 2009). Epistasis analysis indicates that the complex functions in break repair by HR (Lehmann et al. 1995; Verkade et al. 1999; Morishita et al. 2002; McDonald et al. 2003; Onoda et al. 2004; Pebernard et al. 2004, 2006, 2008a; Cost and Cozzarelli 2006; Santa Maria et al. 2007). See Figure 2 for a schematic overview of how Smc5/6 functions in HR. DNA-damage-responsive checkpoint kinases and cell cycle arrest are normally activated in response to UV, MMS, HU, and/or DNA double-strand breaks (DSBs) in budding and fission yeast smc6, smc5, and rad60/esc2 mutants (Verkade et al. 1999; Harvey et al. 2004; Cost and Cozzarelli 2006; Miyabe et al. 2006; Torres-Rosell et al. 2007b; Mankouri et al. 2009). This could be a sign that the Smc5/6 complex is a true repair protein with little effect on the damage checkpoint. However, several studies show that $s m c 5 / 6$ mutated cells reenter the cell cycle without completing repair, leading to chromosome fragmentation during an aberrant mitosis (Verkade et al. 1999; Harvey et al. 2004; Andrews et al. 2005; Ampatzidou et al. 2006; Miyabe et al. 2006) This could suggest that the complex has a role in checkpoint maintenance. However, this in turn is contradicted by the observation that checkpoint maintenance after replication stalling by $\mathrm{HU}$ treatment in smc6-74, smc6-X, rad60-1, and $\Delta e s c 2$ mutants is restored by inhibition of HR through deletion of RAD51 


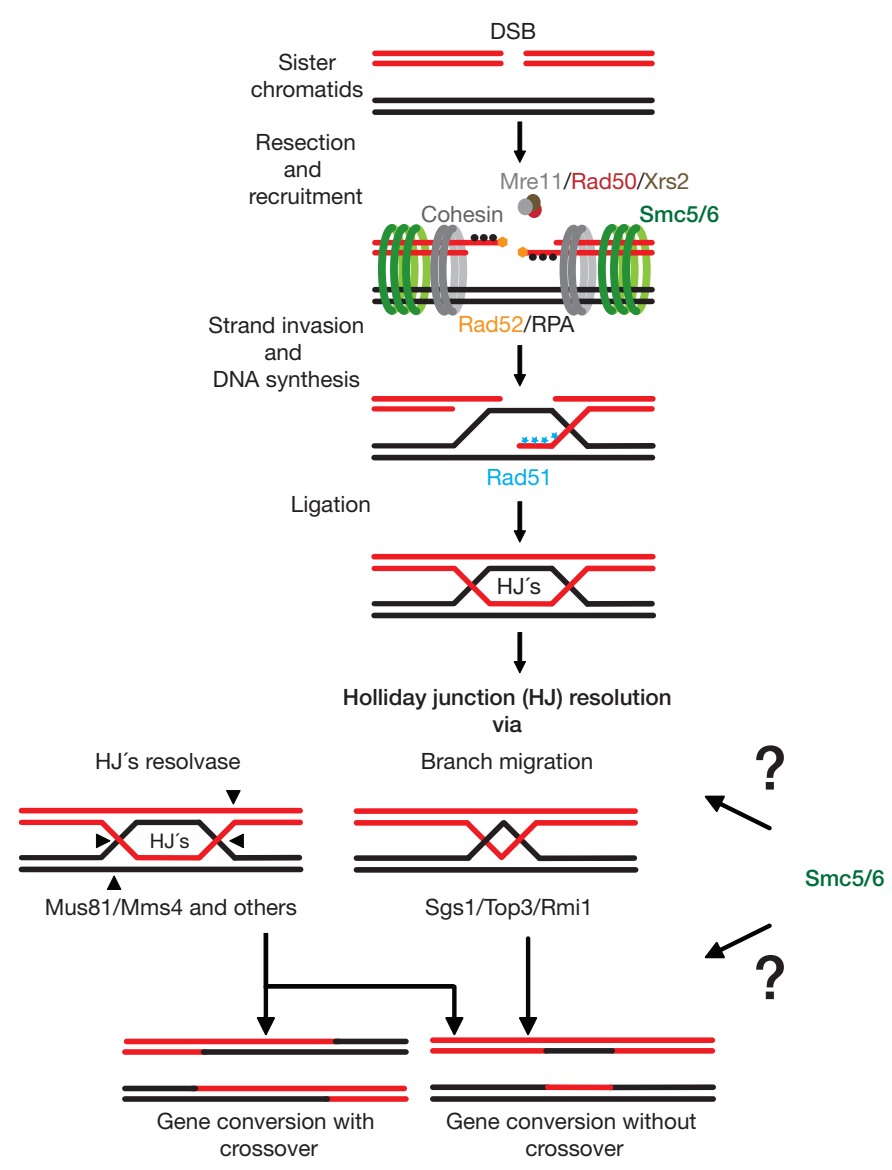

B

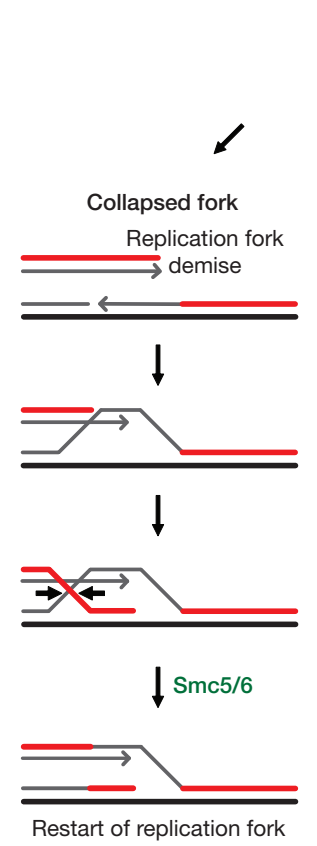

\section{Replication forks}

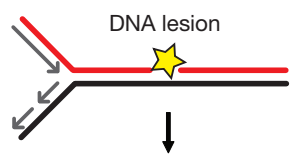

Stable fork

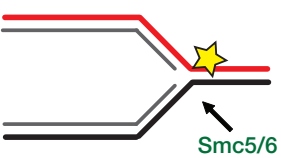

Stabilization of fork
Damage bypass by template switch

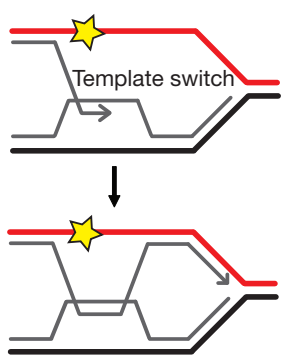

Sgs1/Top3/Rmi1 \Smc5/6

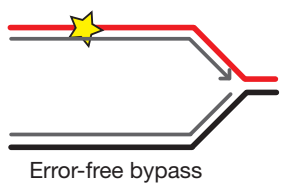

Figure 2. Smc5/6 at DSB and blocked replication forks. The Smc5/6 complex is needed for repair of DSBs and rescue of damaged forks by HR. Shown are simplified views of DSB repair $(A)$ and replication fork rescue $(B)$, focusing on the potential roles of Smc5/6. (A) In DSB repair, Smc5/6 appears to facilitate sister chromatid recombination via cohesin, which is needed to hold the chromatids in close proximity. Without Smc5/6 and cohesin, chromatids will be misaligned, and this can interfere with strand invasion and Holliday junction (HJ) formation. Based on its known function at blocked replication forks, Smc5/6 might also act in parallel with Mus81/Mms4, Sgs1/Top3/Rmi1, and others in the resolution of DSB-induced double HJs. $(B)$ When a replication fork encounters a DNA lesion, it can collapse, which leads to DSB formation and repair by HR. Here, Smc5/6 is thought to work in the resolution of resulting recombination structures. When the fork avoids collapse and remains stable, Smc5/6 has also been proposed to hold the fork in stable recombinationcompetent conformation (Irmisch et al. 2009). Finally, the fork can bypass a lesion via an HR-dependent template switch mechanism. Here, $\mathrm{Smc5} / 6$, as well as Sgs1/Top3/Rmi1, are needed for the resolution of the HR-dependent sister chromatid junctions. 
(Ampatzidou et al. 2006; Miyabe et al. 2006; Mankouri et al. 2009). Moreover, cell survival of smc6-9, smc5-31, smc5-33, and $\Delta n s e 6$ mutants after DNA damage, or after stress due to mutations in other repair proteins, increases in $\Delta$ rad51 or $\Delta$ rad52 genetic backgrounds (Torres-Rosell et al. 2005b; Cost and Cozzarelli 2006; Miyabe et al. 2006; Pebernard et al. 2006; Sollier et al. 2009). This suggests that in the absence of Smc5/6 function, repair by HR is properly initiated and continues to a point at which the checkpoint is tuned down but is not correctly finished. The ensuing resumption of cell cycle progression with incompletely repaired chromosomes leads to a catastrophic anaphase and an increase in cell death. When recombination is inhibited by deletion of genes needed at an early stage of HR, the damage is no longer processed to a checkpoint-invisible structure in $s m c 5 / 6$ mutants. This leads to a prolonged cell cycle delay and allows cells to perform HR-independent repair that increases cell survival. Indeed, an emerging view is that Smc5/6 acts in late steps of HR and is needed for correct resolution of recombination structures that form between sister chromatids at blocked replication forks (see, e.g., Ampatzidou et al. 2006; Miyabe et al. 2006; Pebernard et al. 2006). This is supported by the following observations. First, aberrant Rad51-dependent recombination structures accumulate at MMS-blocked forks in budding yeast $s m c 6$, nse2, and rad60/esc2 mutants (Branzei et al. 2006; Mankouri et al. 2009; Sollier et al. 2009). Second, similar structures are found in the recombination-prone rDNA gene repeats in smc6-9 cells (Torres-Rosell et al. 2005b). Third, cells having a dysfunctional Smc5/6 complex or a defective Rad60/Esc2 protein become more slow growing or die when combined with deletions of the genes coding for the Sgs1 helicase or the Mus81 endonuclease, both involved in the resolution of recombination junctions (Morikawa et al. 2004; Pebernard et al. 2004, 2006, 2008a; TorresRosell et al. 2005b; Miyabe et al. 2006; Raffa et al. 2006; Mankouri et al. 2009; Prudden et al. 2009; Sollier et al. 2009). Mus 81 is also needed for the suppression of fission yeast smc6-74 by Brc1 overexpression (Verkade et al. 1999; Sheedy et al. 2005). Brc1, homologous to Esc4 in budding yeast, encodes a six-BRCT (BRCA1 amino-terminal)-domain protein needed for normal resistance to ionizing irradiation, MMS, HU, and camptothecin (CPT) (Verkade et al. 1999; Rouse 2004; Sheedy et al. 2005; Roberts et al. 2006, 2008). Similar to the Smc5/6 complex, Brc1/Esc4 appears to have a central role for efficient restart of replication after DNA damage, possibly functioning as a docking platform for other repair factors (Rouse 2004; Sheedy et al. 2005; Chin et al. 2006; Roberts et al. 2006, 2008; Lee et al. 2007).

So how does the Smc5/6 complex prevent the accumulation of, and/or resolve, recombination intermediates? Studies of both human and yeast cells suggest that it could work together with cohesin, at least during DSB repair. As stated, cohesin is essential for sister chromatid cohesion, and this tethering of chromatids is essential for correct chromosome segregation. Cohesin is also recruited for DNA breaks, is specifically important for DSB repair by sister chromatid recombination, and appears to facilitate this process by holding the damaged and undamaged sisters in close proximity (Sjögren and Nasmyth 2001; Kim et al. 2002; Strom et al. 2004, 2007; Unal et al. 2004, 2007). The Smc5/6 complex also accumulates at site-specific DSBs and is specifically needed for sister chromatid recombination (De Piccoli et al. 2006; Lindroos et al. 2006; Potts et al. 2006). Furthermore, knockdown of Nse2 or Smc5 inhibits the recruitment of cohesin to a DSB in human cells, and damage-induced chromatid cohesion is defective in budding yeast smc6-56 mutants (Potts et al. 2006; Strom et al. 2007). This could indicate that the Smc5/6 complex, via cohesin, organizes sister chromatids in a way that allows resolution of the recombination intermediates at blocked replication forks. However, in contrast to smc 6 or $n s e 2 \mathrm{mu}-$ tated cells, cohesin malfunction does not lead to an accumulation of aberrant recombination structures during replication in the presence of MMS (Sollier et al. 2009). Thus, the role of Smc5/6 at replication forks appears to be separate from its function in DSB repair outside the $\mathrm{S}$ phase. Another possible function for Smc5/6 could be to position Nse2 (Mms21) at specific chromosomal regions. In 2005, three independent research groups identified Nse2 as a small ubiquitin-like modifier (SUMO) ligase required for DNA repair and able to stimulate sumoylation of itself, Smc5, Smc6, and the repair proteins Yku70 and Trax in vivo (Andrews et al. 2005; Potts and Yu 2005; Zhao and Blobel 2005). Later, Nse2 was also shown to prevent accumulation of aberrant recombination structures at MMSblocked replication forks (Branzei et al. 2006) and to trigger MMS-induced sumoylation and change in nuclear localization of Nse4 (Pebernard et al. 2008b). The Nse2 ligase activity is also essential for stabilization of human telomeres maintained by a so-called alternative lengthening of telomeres (ALT) mechanism that depends on HR (Potts and $\mathrm{Yu}$ 2007). Smc5/6 has been shown to localize to (ALT) telomeres and collapsed replication forks and to change its chromosomal interaction pattern in response to MMS (Torres-Rosell et al. 2005b; Lindroos et al. 2006; Pebernard et al. 2008b). Therefore, it can be envisaged that the role of the non-Nse2 subunits of Smc5/6 is to identify and bind to specific chromosomal structures present at damaged forks and telomeres, thereby placing the SUMO ligase activity where it is needed. If this is true, how sumoylation of the different Nse2 substrates would facilitate sister recombination and prevent formation of aberrant recombination structures remains, however, to be determined.

A very recent publication gives yet another explanation on how Smc5/6 could act at blocked replication forks (Chen et al. 2009). In this study, it is shown that the complex physically interacts with the Mph1 helicase. Moreover, both MMS and HU hypersensitivity and accumulation of recombination structures at MMS-blocked replication forks in smc 6 and nse 2 mutants are suppressed by deletion of MPH1 or by mutations inhibiting its helicase activity (Chen et al. 2009). These investigators propose that Smc5/6 modulates Mph1 to prevent recombination at blocked forks and/or that the complex helps to resolve recombination structures formed by Mph1. Interestingly, $m p h 1 \Delta$ suppresses the lethality of smc 6 and nse2 deletions, allowing slow growth of the double mutants (Chen et al. 2009). This 
indicates that a failure to prevent recombination at naturally occurring fork blocks could be one part of the essential role of the Smc5/6 complex. Is the other part, which remains nonfunctional and, therefore, prevents wild-type growth of smc $6 \Delta m p h 1 \Delta$ and $n s e 2 \Delta m p h 1 \Delta$ mutants, caused by a function in a nonrepair pathway?

\section{THE SMC5/6 COMPLEX: MORE THAN REPAIR?}

The first hint that Smc5/6 does something other than repair by HR is provided by the fact that Smc5, Smc6, Nse1, Nse2, Nse3, and Nse4 are essential for survival of yeast cells. Generally, deletion of genes essential for recombination as such (e.g., RAD51) or involved in the resolution of recombination products (e.g., SGS1) does not lead to lethality. However, HR is essential for survival of higher eukaryotes, so it is possible that the repair function of Smc5/6 is unique in such a way that it also becomes vital for yeast. The isolation of an $S$. pombe separation-offunction mutation in SMC6 that permits cell growth but is defective in repair has also been used as an argument for a nonrepair function of Smc5/6 (Fousteri and Lehmann 2000). Cells with mutations that reduce/inactivate the SUMO ligase activity of Nse2 are also viable but damage hypersensitive (Andrews et al. 2005; Zhao and Blobel 2005). The weak point of these findings, as an indicator of a nonrepair function of Smc5/6, is that the mutants might only be partially defective, with remaining repair activity sustaining survival. Nor does the nuclear morphology of Smc5/6-depleted cells provide a definitive answer as to whether the complex is solely involved in repair. Yeast cells lacking a functional complex accumulate fragmented chromosomes and/or other types of abnormal nuclear structures (Lehmann et al. 1995; Verkade et al. 1999; Fousteri and Lehmann 2000; McDonald et al. 2003). They also activate the DNA-damage checkpoint (Hu et al. 2005; Torres-Rosell et al. 2005a,b). These phenotypes could, in principle, be caused by defective repair of spontaneous damage only, but could also hide a nonrepair function that, when defective, leads to chromosome breakage. When conditional smc5 or smc6 mutants are released into nonpermissive conditions from a $G_{1}$ cell cycle arrest, the damage checkpoint remains inactivated until after the first passage through mitosis (Torres-Rosell et al. 2005b). This could be caused by channeling of spontaneous damage into persistent checkpoint-invisible recombination links between sister chromatids that will lead to chromosome breakage during anaphase and/or cytokinesis. In line with an S-phase - specific role, Smc5/6 is indeed needed during replication for timely cell division (Torres-Rosell et al. 2005b, 2007a; Lindroos et al. 2006). However, no aberrant recombination structures are detected at replication forks in temperature-sensitive smc6 mutants grown at high temperature in the absence of damaging agents (Sollier et al. 2009). This argues for a nonrepair function during $S$ phase that prevents damage formation following chromosome segregation and/or cell division.

So, are there any indications of such a function? Interestingly, although bulk replication seems to be unperturbed in budding yeast $s m c 5 / 6$ mutants as determined by fluorescence-activated cell sorter (FACS) analysis, detailed observations using pulsed field gel electrophoresis (PFGE) of chromosomes isolated from smc6 mutants indicate that Smc5/6 facilitates a late step in replication of both normal and rDNA-containing chromosomes (Lindroos et al. 2006; Torres-Rosell et al. 2007a; Kegel 2011). The late replication delay remains unchanged after deletion of RAD52 or $R A D 51$ genes, supporting the notion that the role of Smc5/6 during unperturbed replication is not to repair DNA damage and/or prevent accumulation of aberrant recombination structures (Torres-Rosell et al. 2007a; Kegel 2011). Additional evidence that Smc5/6 has a nonrepair role during replication comes from investigations of its chromosomal-binding pattern. In budding yeast, the complex is recruited to chromosomes during replication and reaches maximal levels of chromosomal association in $\mathrm{G}_{2} / \mathrm{M}$ cells (Lindroos et al. 2006). In fission yeast and frog, the Smc5/6 chromosomal interaction also increases during replication (Tsuyama et al. 2006; Pebernard et al. 2008b). Because the association is detected in unchallenged cells, and, at least, in budding yeast, it shows no consistent correlation with mapped fragile sites or replication slow zones, it is likely to be independent of DNA damage (Lindroos et al. 2006). Moreover, the Smc5/6 complex is still recruited to $S$. cerevisiae chromosomes in the absence of Mre11, which is needed for the localization of the complex to DNA breaks (Lindroos et al. 2006). Instead, the association is under the control of Scc2, which also regulates cohesin's and condensin's chromosomal interaction during an unchallenged cell cycle (Ciosk et al. 2000; Lindroos et al. 2006; D’Ambrosio et al. 2008).

\section{THE SMC5/6 COMPLEX AND CHROMOSOME TOPOLOGY}

Taken together, it appears that Smc5/6 has a function apart from its role in repair. This facilitates a late step in replication and prevents chromosome fragmentation during passage through mitosis. What could this function be? Intriguingly, when budding yeast Smc5/6 associates with chromosomes during $\mathrm{S}$ phase, it does so in a length-dependent manner; the frequency of interaction sites increases in linear correlation with chromosome length (Lindroos et al. 2006). How could a protein complex be preferentially recruited to longer chromosomes? What mark and/or structure would be determined by chromosome size? Possibly, the reason for the length-dependent association pattern is that Smc5/6 directly or indirectly senses topological stress that accumulates during replication.

For better understanding of this idea, a short introduction of replication-induced topological stress is required (Fig. 3A). This stress is formed as a result of the separation of the two parental strands in the DNA duplex during the passage of the replication fork. The strand separation will cause the DNA ahead of the fork to become overwound or positively supercoiled. To allow complete chromosome duplication, these supercoils must be removed. The main players in this process are enzymes called topoisomerases, which either are type I or type II enzymes (Wang 2002). 

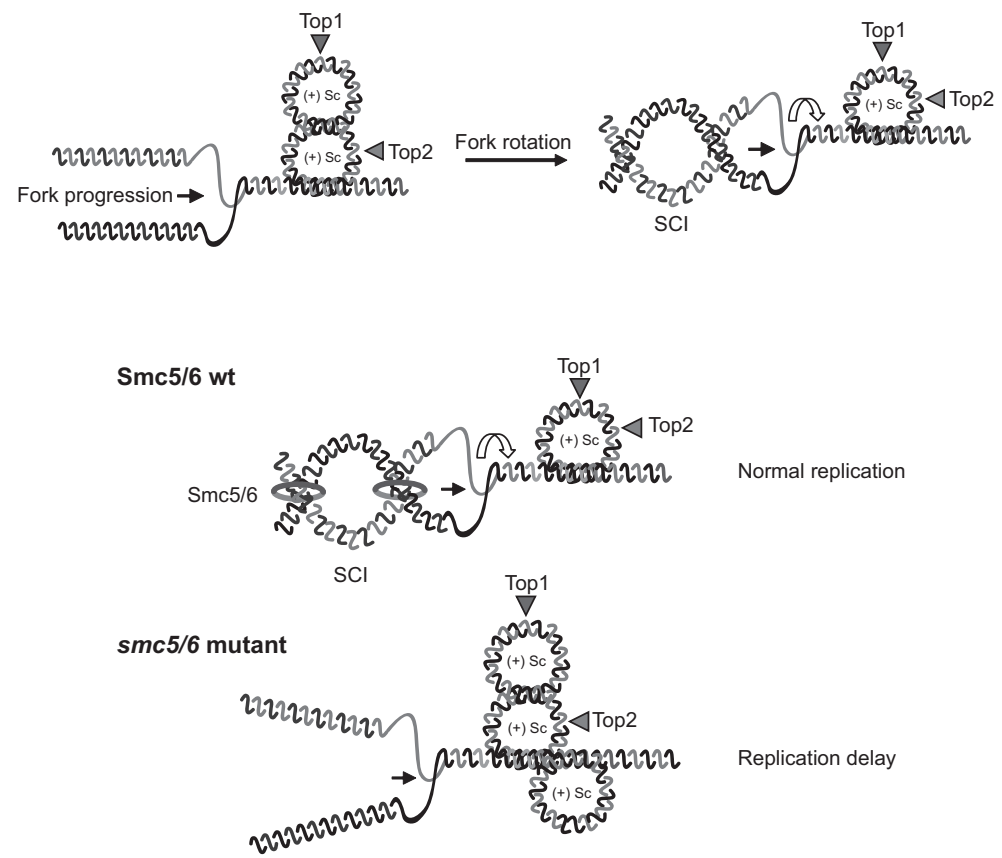

Figure 3. (A) Schematic of replication-induced topological challenges. During replication, the DNA ahead of the advancing replication fork becomes overwound due to separation of the two parental DNA strands. This tightly wound, or positively supercoiled, DNA $(+\mathrm{Sc})$ must be removed to avoid replication inhibition. This is performed by topoisomerases (Top1 and Top2 that release the tension via transient formation of DNA breaks (please see text for details). Another mechanism to avoid accumulation of supercoiled DNA ahead of the fork is to allow the advancing fork to rotate with the turn of the helix. This rotation will lead to sister chromatid intertwinement (SCI) behind. (B) A potential role for Smc5/6 is the resolution of superhelical tension. Smc5/6 sequesters SCIs behind the fork and thereby facilitates the rotation mechanism (Smc5/6 wt). If Smc5/6 is nonfunctional, the rotation mechanism is inhibited; supercoils accumulate ahead of the fork, leading to a replication delay ( $s m c 5 / 6$ mutant).
Type I topoisomerases (e.g., S. cerevisiae Top1 and Top3) relieve superhelical tension by creating single-strand nicks in the DNA, whereas Type II topoisomerases (e.g., S. cerevisiae Top2) transfer one DNA helix through a transient DSB created by the isomerase. Another way to prevent the accumulation of positive supercoils ahead of an advancing replication fork is to allow the replication fork to advance in a rotating manner, following the turn of the DNA helix (Postow et al. 2001; Wang 2002). A rotational movement of the fork will prevent the accumulation of supercoils and instead lead to the formation of sister chromatid intertwinings (or catenations) behind (Fig. 3A). This type of resolution has been proposed to be especially important at the site of replication termination when the two converging forks limit the accesses of topoisomerases.

If the budding yeast Smc5/6 chromosomal-binding pattern is a reflection of higher topological tension on longer chromosomes, it is expected that topoisomerases should be more important for replication of longer chromosomes. And we have indeed found a length-dependent inhibition of late replication in cells lacking functional type I topoisomerases (Kegel 2011). In addition, smc6 and nse2-sumoligase mutants show the same type of replication delay, indicating that Smc5/6 not only senses topological strain but also has a role in its resolution. Moreover, investigations of the association of Smc5/6 with chromosomes with altered topology and plasmid topology assays suggest that Smc5/6 works in the pathway that facilitates fork rotation and transition of positive supercoils ahead of the fork to chromatid intertwinings behind (Fig. 3B) (Kegel 2011). In line with a topological function for the complex, overexpression of Smc6 leads to changes in S. pombe nuclear architecture that are similar to those observed in cells lacking topoisomerases of types I and II (Uemura and Yanagida 1984; Harvey et al. 2004). In addition, the fission yeast smc6-74 mutant is synthetically lethal with temperature- sensitive top2-191 at semipermissive temperatures (Verkade et al. 1999). And budding yeast nse2-sumo-ligase and smc6-56 mutants show synthetic growth defects when combined with mutations in Top2 (Takahashi et al. 2008; A Kegel, unpubl.). Intriguingly, an smc6-9-dependent replication delay in an rDNA-containing budding yeast chromosome is diminished when transcription is inhibited (Torres-Rosell et al. 2007a). Additionally, localization of $S$. pombe Nse4 to transfer RNA (tRNA) genes requires ongoing transcription, and the budding yeast Smc5/6 preferentially interacts with intergenic regions downstream from open reading frames (Lindroos et al. 2006; Pebernard et al. 2008 b). Because the transcription process also induces superhelical tension, it might be that Smc5/6 influences both replication- and transcription-induced topological strain.

Similarly to the function of Smc5/6 during repair, evidence shows that the complex also acts together with cohesin in unchallenged cells. The previously mentioned lethality of the smc6-74 top2-191 mutant appears to be due to a misregulation of cohesin's chromosomal association, because moderate overexpression of the protease separase, which removes cohesin from chromosomes at anaphase, increases survival of the mutated cells (Outwin et al. 2009). Moreover, Nse2 is required for sumoylation of the SMC protein subunits in cohesin (Takahashi et al. 2008). However, the relevance of the functional connections between the two complexes awaits confirmation. This becomes especially clear in light of other recently published data showing that knockdown of Smc5 and Mms21 leads to a cohesion defect in human cells (BehlkeSteinert et al. 2009). Although this is in accordance with the findings that Smc5/6 positively regulates cohesin after DNA damage (Potts et al. 2006; Strom et al. 2007), it is definitively not a phenotype that is expected to be rescued by a factor that removes cohesin from chromosomes (Outwin et al. 2009). Potentially, these discrepancies are due 
to differences in experimental setups. Detailed analysis of chromosome stability and dynamics after complete depletion of each of the Smc5/6 subunits in defined stages of the cell cycle is still lacking and will improve the understanding of the complex. But even without this analysis, current data support a nonrepair topological function of Smc5/6 that allows full replication of chromosomes and prevents chromosome fragmentation during mitosis. This, together with the absence of functional repair, can explain why cells lacking the Smc5/6 complex cannot survive.

\section{ACKNOWLEDGMENTS}

C.S. is a Royal Swedish Academy of Sciences Research Fellow supported by the Knut and Alice Wallenberg Foundation. C.S. and A.K. are also financed by the European Research Council, the Swedish Research Council, the Swedish Cancer Society, Cornell's and Karolinska Institute's research foundations, Vinnova, and the Swedish Foundation for Strategic Research.

\section{REFERENCES}

Ampatzidou E, Irmisch A, O’Connell MJ, Murray JM. 2006. Smc5/6 is required for repair at collapsed replication forks. Mol Cell Biol 26: 9387-9401.

Andrews EA, Palecek J, Sergeant J, Taylor E, Lehmann AR, Watts FZ. 2005. Nse2, a component of the Smc5-6 complex, is a SUMO ligase required for the response to DNA damage. $\mathrm{Mol}$ Cell Biol 25: 185-196.

Arumugam P, Gruber S, Tanaka K, Haering CH, Mechtler K, Nasmyth K. 2003. ATP hydrolysis is required for cohesin's association with chromosomes. Curr Biol 13: 1941-1953.

Behlke-Steinert S, Touat-Todeschini L, Skoufias DA, Margolis RL. 2009. SMC5 and MMS21 are required for chromosome cohesion and mitotic progression. Cell Cycle 8: 2211-2218.

Boddy MN, Shanahan P, McDonald WH, Lopez-Girona A, Noguchi E, Yates IJ, Russell P. 2003. Replication checkpoint kinase Cds1 regulates recombinational repair protein Rad60. Mol Cell Biol 23: 5939-5946.

Branzei D, Sollier J, Liberi G, Zhao X, Maeda D, Seki M, Enomoto T, Ohta K, Foiani M. 2006. Ubc9- and mms21-mediated sumoylation counteracts recombinogenic events at damaged replication forks. Cell 127: 509-522.

Chen YH, Choi K, Szakal B, Arenz J, Duan X, Ye H, Branzei D, Zhao X. 2009. Interplay between the Smc5/6 complex and the Mph1 helicase in recombinational repair. Proc Natl Acad Sci 106: 21252-21257.

Chin JK, Bashkirov VI, Heyer WD, Romesberg FE. 2006. Esc4/Rtt107 and the control of recombination during replication. DNA Repair 5: 618-628.

Ciosk R, Shirayama M, Shevchenko A, Tanaka T, Toth A, Nasmyth K. 2000. Cohesin's binding to chromosomes depends on a separate complex consisting of Scc2 and Scc4 proteins. Mol Cell 5: $243-254$.

Cost GJ, Cozzarelli NR. 2006. Smc5p promotes faithful chromosome transmission and DNA repair in Saccharomyces cerevisiae. Genetics 172: 2185-2200.

Cuperus G, Shore D. 2002. Restoration of silencing in Saccharomyces cerevisiae by tethering of a novel Sir2-interacting protein, Esc8. Genetics 162: 633-645.

D’Ambrosio C, Schmidt CK, Katou Y, Kelly G, Itoh T, Shirahige K, Uhlmann F. 2008. Identification of cis-acting sites for condensin loading onto budding yeast chromosomes. Genes Dev 22: 2215-2227.

De Piccoli G, Cortes-Ledesma F, Ira G, Torres-Rosell J, Uhle S, Farmer S, Hwang JY, Machin F, Ceschia A, McAleenan A, et al. 2006. Smc5-Smc6 mediate DNA double-strand-break repair by promoting sister-chromatid recombination. Nat Cell Biol 8: 1032-1034.

De Piccoli G, Torres-Rosell J, Aragon L. 2009. The unnamed complex: What do we know about Smc5-Smc6? Chromosome Res 17: 251-263.

Dhillon N, Kamakaka RT. 2000. A histone variant, Htzlp, and a Sir1p-like protein, Esc2p, mediate silencing at HMR. Mol Cell 6: 769-780.

Duan X, Sarangi P, Liu X, Rangi GK, Zhao X, Ye H. 2009a. Structural and functional insights into the roles of the Mms21 subunit of the Smc5/6 complex. Mol Cell 35: 657-668.

Duan X, Yang Y, Chen YH, Arenz J, Rangi GK, Zhao X, Ye H. 2009b. Architecture of the Smc5/6 complex of Saccharomyces cerevisiae reveals a unique interaction between the Nse5-6 subcomplex and the hinge regions of Smc5 and Smc6. J Biol Chem 284: 8507-8515.

Fousteri MI, Lehmann AR. 2000. A novel SMC protein complex in Schizosaccharomyces pombe contains the Rad18 DNA repair protein. EMBO J 19: 1691-1702.

Fujioka Y, Kimata Y, Nomaguchi K, Watanabe K, Kohno K. 2002. Identification of a novel non-structural maintenance of chromosomes (SMC) component of the SMC5/SMC6 complex involved in DNA repair. J Biol Chem 277: 21585-21591.

Harvey SH, Sheedy DM, Cuddihy AR, O'Connell MJ. 2004. Coordination of DNA damage responses via the Smc5/Smc6 complex. Mol Cell Biol 24: 662-674.

Hazbun TR, Malmström L, Anderson S, Graczyk BJ, Fox B, Riffle M, Sundin BA, Aranda JD, McDonald WH, Chiu CH, et al. 2003. Assigning function to yeast proteins by integration of technologies. Mol Cell 12: 1353-1365.

Hirano T. 2006. At the heart of the chromosome: SMC proteins in action. Nat Rev Mol Cell Biol 7: 311-322.

$\mathrm{Hu}$ B, Liao C, Millson SH, Mollapour M, Prodromou C, Pearl LH, Piper PW, Panaretou B. 2005. Qri2/Nse4, a component of the essential Smc5/6 DNA repair complex. Mol Microbiol 55: 17351750.

Irmisch A, Ampatzidou E, Mizuno K, O’Connell MJ, Murray JM. 2009. Smc5/6 maintains stalled replication forks in a recombination-competent conformation. EMBO J 28: 144-155.

Kegel A, Betts-Lindroos H, Kanno T, Jeppsson K, Ström L, Katou Y, Itoh T, Shirahige K, Sjögren C. 2011. Chromosome length influences replication-induced topological stress. Nature 471: 392-396.

Kim JS, Krasieva TB, LaMorte V, Taylor AM, Yokomori K. 2002. Specific recruitment of human cohesin to laser-induced DNA damage. J Biol Chem 277: 45149-45153.

Kimura K, Hirano T. 1997. ATP-dependent positive supercoiling of DNA by $13 \mathrm{~S}$ condensin: A biochemical implication for chromosome condensation. Cell 90: 625-634.

Kozak J, West CE, White C, da Costa-Nunes JA, Angelis KJ. 2009. Rapid repair of DNA double strand breaks in Arabidopsis thaliana is dependent on proteins involved in chromosome structure maintenance. DNA Repair 8: 413-419.

Lee K, Nizza S, Hayes T, Bass K, Irmish A, Murray J, O’Connell M. 2007. Brc1-mediated rescue of Smc5/6 deficiency: Requirement for multiple nucleases and a novel Rad18 function. Genetics 175: 1585-1595.

Lehmann AR., Walicka M, Griffiths DJ, Murray JM, Watts FZ, McCready S, Carr AM. 1995. The rad18 gene of Schizosaccharomyces pombe defines a new subgroup of the SMC superfamily involved in DNA repair. Mol Cell Biol 15: 7067-7080.

Lindroos HB, Strom L, Itoh T, Katou Y, Shirahige K, Sjögren C. 2006. Chromosomal association of the Smc5/6 complex reveals that it functions in differently regulated pathways. Mol Cell 22: 755-767.

Mankouri HW, Ngo HP, Hickson ID. 2009. Esc2 and Sgs1 act in functionally distinct branches of the homologous recombination repair pathway in Saccharomyces cerevisiae. Mol Biol Cell 20: 1683-1694.

McDonald WH, Pavlova Y, Yates JR III, Boddy MN. 2003. Novel essential DNA repair proteins Nse1 and Nse2 are subunits of the fission yeast Smc5-Smc6 complex. J Biol Chem 278: 45460-45467.

Mengiste T, Revenkova E, Bechtold N, Paszkowski J. 1999. An 
SMC-like protein is required for efficient homologous recombination in Arabidopsis. EMBO J 18: 4505-4512.

Miyabe I, Morishita T, Hishida T, Yonei S, Shinagawa H. 2006. Rhp51-dependent recombination intermediates that do not generate checkpoint signal are accumulated in Schizosaccharomyces pombe rad60 and smc5/6 mutants after release from replication arrest. Mol Cell Biol 26: 343-353.

Morikawa H, Morishita T, Kawane S, Iwasaki H, Carr AM, Shinagawa H. 2004. Rad62 protein functionally and physically associates with the smc5/smc6 protein complex and is required for chromosome integrity and recombination repair in fission yeast. Mol Cell Biol 24: 9401-9413.

Morishita T, Tsutsui Y, Iwasaki H, Shinagawa H. 2002. The Schizosaccharomyces pombe rad60 gene is essential for repairing double-strand DNA breaks spontaneously occurring during replication and induced by DNA-damaging agents. Mol Cell Biol 22: 3537-3548.

Nasmyth K, Haering CH. 2005. The structure and function of SMC and kleisin complexes. Annu Rev Biochem 74: 595-648.

Onoda F, Takeda M, Seki M, Maeda D, Tajima J, Ui A, Yagi H, Enomoto T. 2004. SMC6 is required for MMS-induced interchromosomal and sister chromatid recombinations in Saccharomyces cerevisiae. DNA Repair 3: 429-439.

Outwin EA, Irmisch A, Murray JM, O’Connell MJ. 2009. Smc5Smc6-dependent removal of cohesin from mitotic chromosomes. Mol Cell Biol 29: 4363-4375.

Palecek J, Vidot S, Feng M, Doherty AJ, Lehmann AR. 2006. The Smc5-Smc6 DNA repair complex. Bridging of the Smc5-Smc6 heads by the KLEISIN, Nse4, and non-Kleisin subunits. $J$ Biol Chem 281: 36952-36959.

Pebernard S, McDonald WH, Pavlova Y, Yates JR III, Boddy MN. 2004. Nse1, Nse2, and a novel subunit of the Smc5-Smc6 complex, Nse3, play a crucial role in meiosis. Mol Biol Cell 15: 4866-4876.

Pebernard S, Wohlschlegel J, McDonald WH, Yates JR III, Boddy MN. 2006. The Nse5-Nse6 dimer mediates DNA repair roles of the Smc5-Smc6 complex. Mol Cell Biol 26: 1617-1630.

Pebernard S, Perry JJ, Tainer JA, Boddy MN. 2008a. Nse1 RINGlike domain supports functions of the Smc5-Smc6 holocomplex in genome stability. Mol Biol Cell 19: 4099-4109.

Pebernard S, Schaffer L, Campbell D, Head SR, Boddy MN. 2008b. Localization of Smc5/6 to centromeres and telomeres requires heterochromatin and $\mathrm{SUMO}$, respectively. EMBO J 27: 3011-3023.

Postow L, Crisona NJ, Peter BJ, Hardy CD, Cozzarelli NR. 2001. Topological challenges to DNA replication: Conformations at the fork. Proc Natl Acad Sci 98: 8219-8226.

Potts PR. 2009. The yin and yang of the MMS21-SMC5/6 SUMO ligase complex in homologous recombination. DNA Repair 8: 499-506.

Potts PR, Yu H. 2005. Human MMS21/NSE2 is a SUMO ligase required for DNA repair. Mol Cell Biol 25: 7021-7032.

Potts PR, Yu H. 2007. The SMC5/6 complex maintains telomere length in ALT cancer cells through SUMOylation of telomerebinding proteins. Nat Struct Mol Biol 14: 581-590.

Potts PR, Porteus MH, Yu H. 2006. Human SMC5/6 complex promotes sister chromatid homologous recombination by recruiting the $\mathrm{SMC} 1 / 3$ cohesin complex to double-strand breaks. EMBO J 25: 3377-3388.

Prudden J, Perry JJ, Arvai AS, Tainer JA, Boddy MN. 2009. Molecular mimicry of SUMO promotes DNA repair. Nat Struct Mol Biol 16: 509-516.

Raffa GD, Wohlschlegel J, Yates JR III, Boddy MN. 2006. SUMObinding motifs mediate the Rad60-dependent response to replicative stress and self-association. J Biol Chem 281: $27973-$ 27981.

Roberts TM, Kobor MS, Bastin-Shanower SA, Ii M, Horte SA, Gin JW, Emili A, Rine J, Brill SJ, Brown GW. 2006. Slx4 regulates DNA damage checkpoint-dependent phosphorylation of the BRCT domain protein Rtt107/Esc4. Mol Biol Cell 17: 539-548.

Roberts TM, Zaidi IW, Vaisica JA, Peter M, Brown GW. 2008. Regulation of rtt107 recruitment to stalled DNA replication forks by the cullin rtt101 and the rtt109 acetyltransferase. Mol
Biol Cell 19: 171-180.

Rouse J. 2004. Esc4p, a new target of Mec1p (ATR), promotes resumption of DNA synthesis after DNA damage. EMBO J 23: 1188-1197.

Santa Maria SR, Gangavarapu V, Johnson RE, Prakash L, Prakash S. 2007. Requirement of Nse1, a subunit of the Smc5-Smc6 complex, for Rad52-dependent postreplication repair of UVdamaged DNA in Saccharomyces cerevisiae. Mol Cell Biol 27: 8409-8418.

Sergeant J, Taylor E, Palecek J, Fousteri M, Andrews EA, Sweeney S, Shinagawa H, Watts FZ, Lehmann AR. 2005. Composition and architecture of the Schizosaccharomyces pombe Rad18 (Smc5-6) complex. Mol Cell Biol 25: 172-184.

Sheedy DM, Dimitrova D, Rankin JK, Bass KL, Lee KM, TapiaAlveal C, Harvey SH, Murray JM, O'Connell MJ. 2005. Brc1mediated DNA repair and damage tolerance. Genetics 171: 457-468.

Sjögren C, Nasmyth K. 2001. Sister chromatid cohesion is required for postreplicative double-strand break repair in Saccharomyces cerevisiae. Curr Biol 11: 991-995.

Sollier J, Driscoll R, Castellucci F, Foiani M, Jackson SP, Branzei D. 2009. The Saccharomyces cerevisiae Esc2 and Smc5-6 proteins promote sister chromatid junction-mediated intra-S repair. Mol Biol Cell 20: 1671-1682.

Strom L, Lindroos HB, Shirahige K, Sjögren C. 2004. Postreplicative recruitment of cohesin to double-strand breaks is required for DNA repair. Mol Cell 16: 1003-1015.

Strom L, Karlsson C, Lindroos HB, Wedahl S, Katou Y, Shirahige K, Sjögren C. 2007. Postreplicative formation of cohesion is required for repair and induced by a single DNA break. Science 317: $242-245$.

Takahashi Y, Dulev S, Liu X, Hiller NJ, Zhao X, Strunnikov A. 2008. Cooperation of sumoylated chromosomal proteins in rDNA maintenance. PLoS Genet 4: e1000215.

Taylor EM, Moghraby JS, Lees JH, Smit B, Moens PB, Lehmann AR. 2001. Characterization of a novel human SMC heterodimer homologous to the Schizosaccharomyces pombe Rad18/Spr18 complex. Mol Biol Cell 12: 1583-1594.

Taylor EM, Copsey AC, Hudson JJ, Vidot S, Lehmann AR. 2008. Identification of the proteins, including MAGEG1, that make up the human SMC5-6 protein complex. Mol Cell Biol 28: 1197-1206.

Terret ME, Sherwood R, Rahman S, Qin J, Jallepalli PV. 2009. Cohesin acetylation speeds the replication fork. Nature 462: 231234.

Torres-Rosell J, Machin F, Aragon L. 2005a. Smc5-Smc6 complex preserves nucleolar integrity in S. cerevisiae. Cell Cycle 4: 868872.

Torres-Rosell J, Machin F, Farmer S, Jarmuz A, Eydmann T, Dalgaard JZ, Aragon L. 2005b. SMC5 and SMC6 genes are required for the segregation of repetitive chromosome regions. Nat Cell Biol 7: 412-419.

Torres-Rosell J, De Piccoli G, Cordon-Preciado V, Farmer S, Jarmuz A, Machin F, Pasero P, Lisby M, Haber JE, Aragon L. 2007a. Anaphase onset before complete DNA replication with intact checkpoint responses. Science 315: 1411-1415.

Torres-Rosell J, Sunjevaric I, De Piccoli G, Sacher M, EckertBoulet N, Reid R, Jentsch S, Rothstein R, Aragon L, Lisby M. 2007b. The Smc5-Smc6 complex and SUMO modification of Rad52 regulates recombinational repair at the ribosomal gene locus. Nat Cell Biol 9: 923-931.

Tsuyama T, Inou K, Seki M, Seki T, Kumata Y, Kobayashi T, Kimura K, Hanaoka F, Enomoto T, Tada S. 2006. Chromatin loading of Smc5/6 is induced by DNA replication but not by DNA double-strand breaks. Biochem Biophys Res Commun 351: 935-939.

Uemura T, Yanagida M. 1984. Isolation of type I and II DNA topoisomerase mutants from fission yeast: Single and double mutants show different phenotypes in cell growth and chromatin organization. EMBO J 3: 1737-1744.

Unal E, Arbel-Eden A, Sattler U, Shroff R, Lichten M, Haber JE, Koshland D. 2004. DNA damage response pathway uses histone modification to assemble a double-strand break-specific co- 
hesin domain. Mol Cell 16: 991-1002.

Unal E, Heidinger-Pauli JM, Koshland D. 2007. DNA doublestrand breaks trigger genome-wide sister-chromatid cohesion through Eco1 (Ctf7). Science 317: 245-248.

Verkade HM, Bugg SJ, Lindsay HD, Carr AM, O’Connell MJ. 1999. Rad18 is required for DNA repair and checkpoint responses in fission yeast. Mol Biol Cell 10: 2905-2918.

Wang JC. 2002 Cellular roles of DNA topoisomerases: A molecular perspective. Nat Rev Mol Cell Biol 3: 430-440.

Weitzer S, Lehane C, Uhlmann F. 2003. A model for ATP hydrolysis-dependent binding of cohesin to DNA. Curr Biol 13: 1930-
1940.

Wendt KS, Peters JM. 2009. How cohesin and CTCF cooperate in regulating gene expression. Chromosome Res 17: 201-214.

Yu Q, Kuzmiak H, Olsen L, Kulkarni A, Fink E, Zou Y, Bi X. 2010. Saccharomyces cerevisiae Esc2p interacts with Sir2p through a small ubiquitin-like modifier (SUMO)-binding motif and regulates transcriptionally silent chromatin in a locus-dependent manner. J Biol Chem 285: 7525-7536.

Zhao X, Blobel G. 2005. A SUMO ligase is part of a nuclear multiprotein complex that affects DNA repair and chromosomal organization. Proc Natl Acad Sci 102: 4777-4782. 


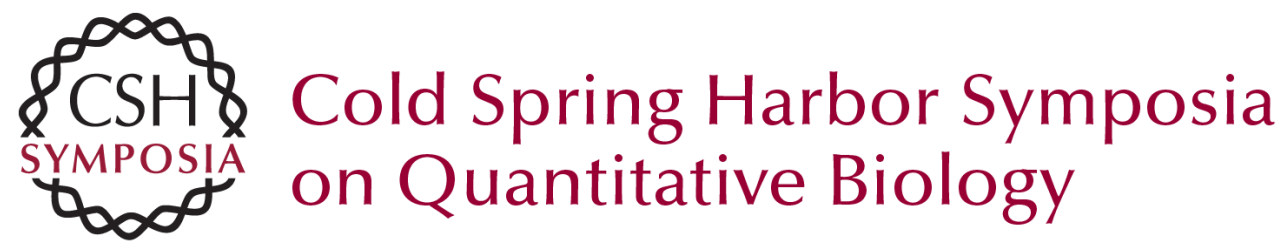

\title{
The Smc5/6 Complex: More Than Repair?
}

\author{
A. Kegel and C. Sjögren
}

Cold Spring Harb Symp Quant Biol 2010 75: 179-187 originally published online April 5, 2011 Access the most recent version at doi:10.1101/sqb.2010.75.047

References This article cites 80 articles, 39 of which can be accessed free at: http://symposium.cshlp.org/content/75/179.full.html\#ref-list-1

License

Email Alerting Receive free email alerts when new articles cite this article - sign up in the box at the Service top right corner of the article or click here.

To subscribe to Cold Spring Harbor Symposia on Quantitative Biology go to: http://symposium.cshlp.org/subscriptions 